Published in final edited form as:

Int J STD AIDS. 2016 June ; 27(7): 531-536. doi:10.1177/0956462415586676.

\title{
Acceptability and feasibility of HIV self-testing among men who have sex with men in Peru and Brazil
}

\author{
Jonathan E Volk ${ }^{1}$, Sheri A Lippman², Beatriz Grinsztejn ${ }^{3}$, Javier R Lama ${ }^{4}$, Nilo M \\ Fernandes $^{3}$, Pedro Gonzales ${ }^{4}$, Nancy A Hessol ${ }^{5}$, and Susan Buchbinder ${ }^{1,6}$ \\ ${ }^{1}$ Bridge HIV, San Francisco Department of Public Health, San Francisco CA, USA \\ ${ }^{2}$ Center for AIDS Prevention Studies, Department of Medicine, University of California, San \\ Francisco, CA, USA \\ ${ }^{3}$ Fundação Oswaldo Cruz, Rio de Janeiro, Brasil \\ ${ }^{4}$ Asociación Civil Impacta Salud y Educación, Lima, Peru \\ ${ }^{5}$ Departments of Clinical Pharmacy and of Medicine, University of California, San Francisco, CA, \\ USA
}

${ }^{6}$ Departments of Medicine, Epidemiology and Biostatistics, University of California, San Francisco, CA, USA

\section{Abstract}

HIV self-testing has the potential to increase testing frequency and uptake. This pilot study assessed the feasibility and acceptability of HIV self-testing in a sample of sexually active men who have sex with men in Peru and Brazil. Participants were trained to use a whole blood rapid HIV self-test and instructed to use the self-test monthly during this three-month study. Test acceptability was measured with self-reported use of the test at the one-month and three-month study visits, and test feasibility was assessed by direct observation of self-test administration at the final three-month visit. A total of 103 participants (52 in Peru and 51 in Brazil) were enrolled, and $86 \%$ completed the three-month study. Nearly all participants reported use of the self-test (97\% at one-month and $98 \%$ at three-month visit), and all participants correctly interpreted the selfadministered test results when observed using the test at the final study visit. HIV self-testing with a blood-based assay was highly acceptable and feasible. HIV self-testing may have the potential to increase testing frequency and to reach high-risk men who have sex with men not currently accessing HIV-testing services.

Reprints and permissions: sagepub.co.uk/journalsPermissions.nav

Corresponding author: Jonathan E Volk, 25 Van Ness Avenue, Suite 100, San Francisco, CA 94102-6033, USA.

jvolk@stanfordalumni.org.

Declaration of Conflicting Interests

The authors declared no potential conflicts of interest with respect to the research, authorship, and/or publication of this article. Chembio Diagnostic Systems, Inc. donated the test kits, but had no participation in study design, implementation, analyses or drafting of this manuscript. The views expressed herein do not necessarily reflect the official policies of the City and County of San Francisco; nor does mention of the San Francisco Department of Public Health imply its endorsement. 


\section{Keywords}

HIV; viral disease; homosexual; diagnosis; South America; screening

\section{Introduction}

In Peru and Brazil, men who have sex with men (MSM) have the highest HIV prevalence and incidence compared to other HIV transmission groups. ${ }^{1-3}$ HIV prevention strategies currently available are inadequate to control the spread of new infections among MSM, thus new interventions are needed. Vital to any prevention strategy is HIV testing, the primary gateway into HIV care and treatment. Studies have suggested that a substantial proportion of new infections come from persons unaware of their HIV infection, ${ }^{4,5}$ and that increased testing alone could reduce new infections by as much as one-third in one year under optimal circumstances. ${ }^{6}$ HIV self-testing kits, now available over the counter (OTC) in many Western countries, have the potential to increase the proportion of people tested, increase testing frequency, and encourage earlier detection of HIV and thus earlier treatment.

Prior research in Brazil has documented the feasibility and acceptability of home-based selfcollection of biologic specimens for other sexually transmitted diseases, ${ }^{7}$ as well as hypothetical interest in HIV self-testing. ${ }^{8}$ Recently, high-risk MSM and transgender women in Peru reported a relatively low rate of willingness to use home-based HIV tests for regular HIV testing (Lama JR, personal communication); however, there are no published studies to date documenting experiences with HIV self-testing in South America. We conducted a three-month longitudinal pilot study to evaluate the feasibility and acceptability of HIV home testing among MSM in Peru and Brazil using a fingerstick whole blood assay. We chose a whole blood rather than an oral secretion home-based test because of its increased sensitivity during acute infection. We hypothesised that feasibility and acceptability for HIV self-testing would be high in a sample of sexually active MSM.

\section{Methods}

Study sample

Participants were enrolled at one study site in Rio de Janeiro, Brazil and two sites in Lima, Peru. These sites were selected because of their participation in the Prevention Umbrella for MSM in the Americas, a larger multi-site international study designed to explore optimal combination HIV prevention strategies for MSM. Recruitment occurred through community outreach and online and print advertisements. Participants were eligible for inclusion if they were HIV-uninfected, male sex at birth and currently identified as male, at least 18 years of age, a resident of Lima or Rio de Janeiro, able to read in Spanish or Portuguese, and willing to provide informed consent. To target MSM at high risk for HIV-acquisition, men who reported anal sex with two or more male partners or any unprotected anal sex with male partners in the preceding six months were eligible for inclusion. HIV status was confirmed with a rapid HIV test prior to enrollment. ${ }^{9,10}$ 


\section{Study design}

This three-month, longitudinal study included three in-person study visits. Following consent at the initial visit, participants completed a Computer Assisted Self-Interview (CASI) to assess demographics, health care utilisation, HIV risk behaviours, and HIV testing history and attitudes. Training on the use of the test kit was then conducted by a member of the study staff and included direct observation of participants using the Clearview COMPLETE HIV-1/2 Assay prior to distribution of the whole blood rapid HIV self-test. This assay was chosen because of its high sensitivity and specificity. Participants were instructed to use the kit prior to their one-month, in-person follow-up visit. All test kits had written instructions that included pictures, pre- and post-test counselling materials, a list of local HIV/AIDS resources, and condoms. Participants were asked to contact a 24-h telephone support line for any positive or invalid test results, procedural questions, technical support, counselling, or to request new test kits. Monthly SMS text message and email reminders to use the self-test were provided for participants with mobile phones or e-mail access.

At the one-month study visit, participants completed a CASI questionnaire about their selftesting experience, including questions about ease of use, confidence in results, and willingness to test again. Participants were given two additional kits and instructed to test monthly. Monthly testing was used to maximise participant experience using the test device. At the three-month final study visit, a CASI was used to assess test acceptability, as well as history, attitudes, and barriers to home testing and HIV risk behaviours. Participants were asked to use the self-test independently during this final visit while being directly observed and assessed by a trained study staff member; participants did not receive assistance from study staff while conducting the test.

\section{Measures and statistical analyses}

Our primary dependent variables were testing acceptability and testing feasibility.

Acceptability was measured with the self-reported use of the test at the one-month and three-month study visits. Self-testing feasibility was assessed at the three-month visit; participants were classified as using the test incorrectly if errors were made during self-test administration that resulted in an inaccurate result. Questions regarding use of the test kit and trust in the results were asked using a 4-point Likert scale and made into dichotomous variables. Willingness to recommend the test to a friend and desire to use the test in the future were asked as yes/no questions. Statistical analyses were performed using SAS $^{\circledR}$ software version 9.3.

\section{Results}

One hundred and three participants enrolled (52 in Peru and 51 in Brazil), 92 (89\%) returned for the one-month visit, and $89(86 \%)$ completed the three-month study visit. At study entry, about half (49\%) of the study participants were 25 years old or younger, and $25 \%$ had 10 years of education or fewer (Table 1). Forty-six participants (45\%) had not been tested for HIV in the preceding year, and $16 \%$ had never been tested for HIV prior to study participation. In the three months prior to study enrolment, 59\% reported anal sex without a 
condom, $17 \%$ were diagnosed with a sexually transmitted infection, and $40 \%$ reported sexual activity while under the influence of alcohol. A history of exchange sex was also common (28\%).

Test acceptability was high, with 98\% (90/92) reporting use of the self-test at the one-month visit, and 97\% (86/89) reporting use of the kit at the final three-month study visit. Reasons cited for not using the test included loss of the test, forgetfulness, lack of time, lack of perceived risk for HIV, and desire to avoid using the lancet to draw blood. At the final study visit, 92\% (82/89) of study participants reported feeling comfortable collecting their own blood specimens, 91\% (81/89) found it easy to obtain blood, and 98\% (87/89) described test interpretation as straight forward; 99\% (88/89) reported that they trust future results if the test were used again. All participants (89/89) noted that they would recommend the test to a friend, and 99\% (88/89) reported that they would use the kit themselves were they available in the future. No participants reported giving the tests to people outside of the study.

A large majority ( $86 \%$ ) of the tests were completed in participants' homes, and a few were used at the homes of partners (7\%), friends (3\%), and relatives (2\%). A few kits were also used at places of work (3\%). While most participants reported performing the test alone (68\%), some also had partners or spouses $(15 \%)$, other relatives $(8 \%)$, or friends $(7 \%)$ with them.

Two new HIV infections were diagnosed during the study. One participant in Brazil had a positive result while learning to use the self-test kit during the initial study visit; this individual was linked to care and did not complete the remainder of the study. In Peru, a second participant noted positive results with the home self-test between his one-month and three-month study visits. This positive test was not reported to the study team until the threemonth visit, five days after his initial positive self-test result. The participant was successfully linked to care. The 24-h telephone support line was only used for logistical questions such as rescheduling study appointments or requesting additional self-test kits.

All participants interpreted the results correctly when observed using the self-test at the final study visit. While no errors were made that impacted test results, 28 (31\%) participants made minor errors during testing: 20 (22\%) did not clean their finger with alcohol and/or allow time for the alcohol to dry, $12(13 \%)$ did not dispose of the first drop of blood as recommended by the test instructions, and $10(11 \%)$ had difficulty pushing the test device into the test holder.

\section{Discussion}

In this study of sexually active HIV-uninfected MSM in Peru and Brazil, HIV self-testing with a blood-based assay was both highly acceptable and feasible. Nearly all participants reported use of the self-test, very few participants had concerns with fingerstick blood collection, and all participants correctly interpreted the self-administered test results. Many participants cited lack of time, privacy concerns, and stigma/discrimination as reasons for not getting tested in the year prior to study participation. Importantly, HIV self-testing may 
mitigate these frequently cited barriers by eliminating travel time and waiting at clinics as well as concerns surrounding confidentiality.

Our study findings are consistent with a community-based study of HIV self-testing with oral fluid in Malawi that found high levels of test acceptability and a 99\% concordance between the self and laboratory performed tests. ${ }^{11}$ In contrast, however, another study that included both HIV-infected and uninfected men and women in Singapore using a different blood-based self-test found that $85 \%$ of study participants did not perform all test steps correctly. Consequently, the majority (56\%) of participants had invalid results. ${ }^{12}$ This result may be the result of differences in test characteristics and ease of use, study population, or the level of test instruction included in this study.

Several limitations should be acknowledged when interpreting these results. First, while efforts were made to obtain a diverse sample of MSM in regard to age, education, and ethnicity, our sample did not include MSM from outside major urban areas in Peru and Brazil or MSM who reported lower risk sexual behaviours for HIV-acquisition. Second, we likely also missed MSM who may be fearful of HIV testing, who may not self-identify as gay or bisexual, and who are homeless, marginally housed, or youth living with parents, thus further limiting the generalisability of our findings. Consequently, stigma and discrimination may play important roles in test acceptability and feasibility that were not observed in our study. Third, it is uncertain how many participants were invited to participate in this study and refused (a measure of uptake), thus the response rate is unknown and the refusal rate may have introduced a self-selection bias. In addition, social desirability bias may have resulted in the over-reporting of test use. Finally, our results likely overestimate test acceptability and feasibility, as real-world implementation of HIV self-testing will likely not involve SMS and email reminders or in-person test education and feedback. Cost of the test also has potential to limit uptake.

The U.S. Food and Drug Administration approved the in-home, oral fluid OraQuick test in the United States in 2012. ${ }^{13}$ However, a recent systematic review and meta-analysis concluded that OraQuick In-Home HIV Test has lower sensitivity and positive-predictive value as compared to tests that used fingerstick blood. ${ }^{14}$ In fact, a model of HIV transmission dynamics suggested that replacing clinic-based testing with the OraQuick InHome HIV Test may result in increased HIV prevalence in some populations because of the relative decrease in test sensitivity soon after infection. ${ }^{15}$ This increased sensitivity for early infection would be critical if home self-testing were integrated into an HIV pre-exposure prophylaxis delivery program.

We observed high feasibility, acceptability, and interest in HIV self-testing with a bloodbased assay among at-risk MSM study participants in Peru and Brazil. Our study findings make fingerstick whole blood self-testing an appealing alternative to oral fluid in Peru and Brazil, where OTC self-test kits are not yet being considered by regulatory agencies. Access to low-cost, affordable HIV self-testing offers potential both to increase testing frequency and to reach MSM not currently accessing HIV-testing services. Ongoing efforts will be needed to confirm self-test accuracy outside of a trial setting, and to ensure individuals with positive test results are successfully linked to care for confirmatory testing and treatment. 


\section{Acknowledgments}

The authors would like to acknowledge all the participants in the study as well as field crew members such as peer outreach workers and research staff at study sites, without whom this study would not have been possible. The CASI questionnaire used in this study was developed using a homebrew software created at IMPACTA.

\section{Funding}

The authors disclosed receipt of the following financial support for the research, authorship, and/or publication of this article: This work is supported by NIH grant R01-AI083060. Dr. J.E.V. received support from the Traineeship in AIDS Prevention Studies T32 post-doctoral fellowship (MH-19105-23) from the National Institutes of Mental Health of the U.S. Public Health Service.

\section{References}

1. Alarcón JO, Pun M, Gutiérrez C, et al. Estimation and analysis of HIV incidence in the adult population in Peru: results of application of the MoT mathematical model. Rev Peru Med Exp Salud Publica. 2012; 29:452-460. [PubMed: 23338629]

2. Ministry of Health. National plan for coping with AIDS and STD epidemic among gay men, MSM and transvestites. Brasília: Secretariat of Health Surveillance - Department of STD, AIDS and Viral Hepatitis; 2007.

3. Doctoral Thesis. Federal University of Ceará; Fortaleza: 2012. Respondent driven sampling (RDS) applied to male population who have sex with men in Brazil. Rosa Maria Salani Mota

4. Pinkerton SD, Holtgrave DR, Galletly CL. Infections prevented by increasing HIV serostatus awareness in the United States, 2001 to 2004. J Acquir Immune Defic Syndr. 2008; 47:354-357. [PubMed: 18176322]

5. Marks G, Crepaz N, Janssen RS. Estimating sexual transmission of HIV from persons aware and unaware that they are infected with the virus in the USA. AIDS. 2006; 20:1447-1450. [PubMed: 16791020]

6. Holtgrave DR, Pinkerton SD. Can increasing awareness of HIV seropositivity reduce infections by 50\% in the United States? J Acquir Immune Defic Syndr. 2007; 44:360-363. [PubMed: 17159653]

7. Lippman SA, Jones HE, Luppi CG, et al. Home-based self-sampling and self-testing for sexually transmitted infections: acceptability and feasibility alternatives to provider- based screening in lowincome women in São Paulo, Brazil. Sex Trans Dis. 2007; 34:421-428.

8. Lippman SA, Périssé ARS, Veloso VG, et al. Acceptability of self-conducted HIV testing among MSM in Brazil - data from an on-line survey. Cadernos de Saúde Publica. 2014; 30:724-734. [PubMed: 24896048]

9. Ministry of Health. Technical guideline for HIV infection diagnosis. Brasília: Secretariat of Health Surveillance - Department of STD, AIDS and Viral Hepatitis; 2013.

10. Ministerio de Salud. a con Infeccion por el Virus de Inmunodeficiencia Humana. Peru: Norma Tecnica de Salud para la Atencion Integral del Adulto; 2012.

11. Choko AT, Desmond N, Webb EL, et al. The uptake and accuracy of oral kits for HIV self-testing in high HIV prevalence setting: a cross-sectional feasibility study in Blantyre, Malawi. PLoS Med. 2011; 8:e1001102. [PubMed: 21990966]

12. Lee VJ, Tan SC, Earnest A, et al. User acceptability and feasibility of self-testing with HIV rapid tests. J Acquir Immune Defic Syndr. 2007; 45:449-453. [PubMed: 17554213]

13. OraSure Receives FDA approval of OraQuick ${ }^{\circledR}$ In-Home Test. MarketWatch. Jul 3. 2012 http:// www.market-watch.com/story/orasure-receives-fda-approval-of-oraquickr-in-home-hivtest-2012-07-03

14. Pai NP, Balram B, Shivkumar S, et al. Head-to-head comparison of accuracy of a rapid point-ofcare HIV test with oral versus whole-blood specimens: a systemic review and metanalysis. Lancet Infect Dis. 2012; 12:373-380. [PubMed: 22277215]

15. Katz DA, Cassels SL, Stekler JD. Replacing clinic-based tests with home-use tests may increase HIV prevalence among seattle men who have sex with men: evidence from a mathematical model. Sex Trans Dis. 2014; 41:2-9. 


\section{Table 1}

Baseline demographics, HIV testing history and attitudes, and HIV risk behaviours $(N=103)$.

\begin{tabular}{|c|c|c|c|}
\hline & Peru $n=52(\%)$ & Brazil $n=51(\%)$ & Total $N=103$ \\
\hline \multicolumn{4}{|l|}{ Age } \\
\hline $18-20$ & $12(23)$ & $8(16)$ & $20(19)$ \\
\hline $21-25$ & $20(38)$ & $10(20)$ & $30(29)$ \\
\hline$>25$ years old & $20(38)$ & $33(65)$ & $53(51)$ \\
\hline \multicolumn{4}{|l|}{ Race/ethnicity } \\
\hline Mestizo/Pardo/Mixed race & $40(77)$ & $18(35)$ & $58(56)$ \\
\hline White & $8(15)$ & $19(37)$ & $27(26)$ \\
\hline Black & $3(6)$ & $13(25)$ & $16(16)$ \\
\hline Indigenous/other & $1(2)$ & $1(2)$ & $2(2)$ \\
\hline \multicolumn{4}{|l|}{ Residence } \\
\hline Own house or apartment & $26(50)$ & $30(59)$ & $56(54)$ \\
\hline At a rented apartment/room & $13(25)$ & $14(27)$ & $27(26)$ \\
\hline At a relatives or friend's house & $12(23)$ & $7(14)$ & $19(18)$ \\
\hline \multicolumn{4}{|l|}{ Live alone } \\
\hline Yes & $8(16)$ & $9(18)$ & $17(17)$ \\
\hline \multicolumn{4}{|l|}{ Work status } \\
\hline Full-time job & $28(54)$ & $25(49)$ & $53(51)$ \\
\hline Part-time/hourly work & $12(23)$ & $6(12)$ & $18(17)$ \\
\hline Student & $8(15)$ & $3(6)$ & $11(11)$ \\
\hline Unemployed/other & $4(8)$ & $17(33)$ & $21(20)$ \\
\hline \multicolumn{4}{|l|}{ Years of school } \\
\hline $1-5$ & $9(18)$ & $4(8)$ & $13(13)$ \\
\hline $6-10$ & $5(10)$ & $8(16)$ & $13(13)$ \\
\hline $11-12$ & $11(22)$ & $16(31)$ & $27(27)$ \\
\hline $13-16$ & $18(37)$ & $13(25)$ & $31(31)$ \\
\hline$>16$ & $6(12)$ & $10(20)$ & $16(16)$ \\
\hline \multicolumn{4}{|l|}{ Marital status } \\
\hline Single/separated/divorced & $49(94)$ & $38(75)$ & $87(84)$ \\
\hline Married or living together & $3(6)$ & $12(24)$ & $15(15)$ \\
\hline \multicolumn{4}{|l|}{ Sexual orientation } \\
\hline Homosexual/Gay & $36(69)$ & $42(82)$ & $78(76)$ \\
\hline Bisexual & $14(27)$ & $7(14)$ & $21(20)$ \\
\hline Other & $2(4)$ & $2(4)$ & $4(4)$ \\
\hline \multicolumn{4}{|l|}{ Time since last HIV test } \\
\hline In the last 3 months & $8(16)$ & $16(31)$ & $24(24)$ \\
\hline $3-6$ months & $6(12)$ & $9(18)$ & $15(15)$ \\
\hline 6-12 month & $9(18)$ & $7(14)$ & $16(16)$ \\
\hline $1-2$ years & $12(24)$ & $5(10)$ & $17(17)$ \\
\hline$>2$ years & 7 (14) & $6(12)$ & $13(13)$ \\
\hline
\end{tabular}




\begin{tabular}{|c|c|c|c|}
\hline & Peru $n=52(\%)$ & Brazil $n=51(\%)$ & Total $N=103$ \\
\hline Never tested & $8(16)$ & $8(16)$ & $16(16)$ \\
\hline \multicolumn{4}{|l|}{ Reasons cited for not testing/delaying testing: } \\
\hline \multicolumn{4}{|l|}{ Confidentiality/Stigma/Discrimination } \\
\hline Didn't want others to know getting testing & $9(17)$ & $7(14)$ & $16(15)$ \\
\hline Didn't want health records to show results & $6(11)$ & $0(0)$ & $6(6)$ \\
\hline Concern for stigma/discrimination from health centre & $8(15)$ & $1(2)$ & $9(9)$ \\
\hline Concern for stigma/discrimination from community & $9(17)$ & $3(6)$ & $12(12)$ \\
\hline Worried about being seen at testing centre & $10(19)$ & $2(4)$ & $12(12)$ \\
\hline Didn't want others to know the results & $8(15)$ & $4(8)$ & $12(12)$ \\
\hline \multicolumn{4}{|l|}{ Convenience } \\
\hline Didn't have time & $21(40)$ & $13(25)$ & $34(33)$ \\
\hline Location inconvenient for testing & $7(13)$ & $3(6)$ & $10(10)$ \\
\hline \multicolumn{4}{|l|}{ Other reasons } \\
\hline Negative prior experiences with HIV test & $3(6)$ & $2(4)$ & $5(5)$ \\
\hline Didn't want to receive HIV counselling & $3(6)$ & $1(2)$ & $4(4)$ \\
\hline Didn't feel comfortable with staff at clinic & $4(8)$ & $1(2)$ & $5(5)$ \\
\hline Didn't feel at risk for HIV & $11(21)$ & $5(10)$ & $16(15)$ \\
\hline Didn't want to know results & $7(13)$ & $3(6)$ & $10(10)$ \\
\hline Too expensive & $4(8)$ & $0(0)$ & $4(4)$ \\
\hline \multicolumn{4}{|l|}{ Condoms with male partners in the last 3 months } \\
\hline Never & $9(18)$ & $6(12)$ & $15(15)$ \\
\hline Less than half the time & $8(16)$ & $10(20)$ & $18(18)$ \\
\hline About half the time & $3(6)$ & $2(4)$ & $5(5)$ \\
\hline Over half the time & $10(20)$ & $11(22)$ & $21(21)$ \\
\hline Always & $20(39)$ & $22(43)$ & $42(41)$ \\
\hline \multicolumn{4}{|l|}{ HIV-positive or unknown HIV status partner in the last 3 months } \\
\hline Yes & $33(63)$ & $38(75)$ & $71(69)$ \\
\hline \multicolumn{4}{|l|}{ STI diagnoses in the last 3 months } \\
\hline Yes & $10(19)$ & $7(14)$ & $17(17)$ \\
\hline \multicolumn{4}{|l|}{ Sex while drunk in the last 3 months } \\
\hline Yes & $22(45)$ & $19(35)$ & $40(40)$ \\
\hline \multicolumn{4}{|l|}{ Ever received money or gifts in exchange for sex } \\
\hline Yes & $11(21)$ & $18(35)$ & $29(28)$ \\
\hline
\end{tabular}

STI: sexually transmitted infection. Percentages may not add to $100 \%$ because of rounding. 